\title{
Hacia una teoría de la conciencia expresiva. La propuesta fichteana en el $\S 1$ de la GWL
}

Gustavo Macedo Rodríguez

\section{OpenEdition}

Journals

Edición electrónica

URL: http://journals.openedition.org/ref/326

DOI: $10.4000 /$ ref.326

ISSN: 2258-014X

Editor

EuroPhilosophie Editions

Referencia electrónica

Gustavo Macedo Rodríguez, " Hacia una teoría de la conciencia expresiva. La propuesta fichteana en el § 1 de la GWL », Revista de Estud(i)os sobre Fichte [En línea], 1 | 2010, Publicado el 14 junio 2010 consultado el 08 septiembre 2020. URL : http://journals.openedition.org/ref/326 ; DOI : https:// doi.org/10.4000/ref.326

Este documento fue generado automáticamente el 8 septiembre 2020.

(c) EuroPhilosophie 


\title{
Hacia una teoría de la conciencia expresiva. La propuesta fichteana en el $§ 1$ de la GWL
}

\author{
Gustavo Macedo Rodríguez
}

\section{I}

1 En la obra de Fichte encontramos una continuidad del proyecto kantiano y al mismo tiempo una reformulación del mismo. Tanto en el GWL como también en la WLnm, encontramos los primeros intentos de Fichte para fundar una doctrina de la ciencia (Wissenschaftslehre) capaz de fundamentar el conocimiento humano. De lo que se trata es de desarrollar una filosofía que pueda ser caracterizada a partir un programa encaminado a fundamentar una teoría del saber humano. Estos textos rescatan, por un lado, las primeras intuiciones de Fichte en su intento por refutar el escepticismo epistémico, ${ }^{1}$ además de presentar, por otro, los fundamentos de su método filosófico, el cual, dicho de manera breve, es caracterizado como una sistematización sintética del saber. Dicho en otros términos, un sistema filosófico que funda la posibilidad misma del saber humano en cuanto resultado e incorpora dentro del sistema, de manera original, la función normativa del Yo en cuanto expresión, esto es, como resultado de acciones concretas en el marco de un sistema. Dicho de otra manera: la WL puede ser vista como un sistema que resuelve contraposiciones o contradicciones entre la acción misma del sujeto y el producto de su actuar logrando de este modo establecer una fundamentación del saber humano.

2 En lo que sigue sólo me ocuparé de una función específica adscrita al Yo en el $§ 1 \mathrm{GWL}$, la cual podría ser caracterizada como expresiva y que remite a la auto-posición indeterminada del Yo, la cual es necesaria en el desarrollo del proyecto de la WL. En esta función es mostrado un carácter filosóficamente radical de la subjetividad. Paralelamente es posible realizar una reconstrucción de los distintos tránsitos desde el principio de identidad $(\mathrm{A}=\mathrm{A})$ a la cristalización del Yo como agente de conocimiento 
(Ich bin), pero esta reconstrucción tendrá que ser tema de otro ensayo. De este modo, el objetivo principal aquí será mostrar la importancia de la autoexpresión del Yo en el desarrollo de la teoría de la subjetividad fichteana, para lo cual, no obstante, los resultados de los tránsitos antes mencionados deberán ser aceptados como supuestos. ${ }^{2}$ No debe esperarse, por ello, un análisis inmanente de la obra fichteana, sino una problematización de la noción de 'expresividad' a partir del $§ 1$ de GWL. ${ }^{3}$

\section{II}

La primera tarea que debe ocuparnos es la clarificación del término 'expresividad' o 'carácter expresivo' del Yo. Cuando se habla del carácter expresivo del Yo, es referida una capacidad subjetiva que explica, por un lado, lo característico de lo conceptual y, de otro, cómo tienen lugar acciones como la auto-posición o indeterminación del Yo. La filosofía fichteana intenta explicar la unidad de lo teórico y lo práctico desde la convicción de la supremacía de la razón práctica sobre la teórica, en cuanto concibe dicha unidad no como una mera contemplación o abstracción, sino como el resultado de una acción puesta por el Yo en la cual sujeto y objeto son uno y lo mismo (Tathandlung). ${ }^{4}$ Fichte pretende demostrar de manera apodíctica la posibilidad de una autoconciencia libre, que se materializa por medio de la acción misma del autoponerse.

4 En esto consiste la novedad de su concepción de subjetividad: mostrar la unidad de lo subjetivo y objetivo desde la posibilidad del autoconocimiento, es decír, desde el conocimiento de las facultades humanas en su propia esfera. Fichte asume que es posible postular un Yo que se determine a sí mismo y éste es precisamente el sentido del carácter expresivo del Yo, al cual el presente artículo dedica especial interés. Además, Fichte acentúa que esta auto-posición del Yo no es simplemente una condición de posibilidad del Yo epistémico (tomado como sujeto), sino que el primero se autoconstituye en la infinitud, esto es, representa una función subjetiva que sólo adquiere sentido en cuanto acto de una auto-posición, en la que sujeto y objeto son intuidos como siendo uno y lo mismo. De esta manera puede ser entendida la afirmación fichteana de que el Yo tiene que ponerse para sí mismo y no para una inteligencia externa a él. (cf. GA I, 2, $406 \mathrm{GWL}$ ) Lo que habría que acentuar es, pues, un postulado de la reflexión, que metodológicamente se desmarca de una investigación empírica y que constituye su propio objeto. De ahí la necesidad de delinear las diferencias específicas del proyecto Fichteano frente a sus antecesores, ya que su proyecto no versa sobre la facultad de juzgar o sobre proposiciones empíricas, sino más específicamente, sobre las condiciones de posibilidad del pensamiento, del querer, del desear. (Cf. GA IV, 3, 328 WLnm) Puesto que el autoconocimiento mismo consiste en sus autoexpresiones, a saber, en la capacidad humana de reflexionar sobre sus propias condiciones (autoexpresión), debe ser intensificada la investigación de manera inmanente o genética, pues el sujeto, visto como objeto de su propio pensamiento, tiene lugar en un plano actual y potencial. ${ }^{5}$ Cabe aquí la aclaración de que la función del Yo dentro de este intento programático se justifica porque es concebida como una mera actividad y no como un hecho del mundo o una sustancia en sentido pre-crítico, esto es, en cuanto substrato.

Por ello, una de las primeras empresas del proyecto fichteano, la cual es desarrollada en el $§ 1$ de GWL, es caracterizar y fundamentar este principio incondicionado, a partir del 
cual puedan derivarse, con certeza, nuestras posteriores inferencias. Fichte se pregunta por la procedencia de la certeza de las proposiciones que darán lugar a un sistema de las ciencias en general o, dicho con sus propios términos, de la ciencia de las ciencias. Según Fichte, tendríamos que preguntarnos en primer lugar: ¿cómo es dicha ciencia posible? Adicionalmente, habría que responder también a la siguiente pregunta de D. Henrich, la cual traza el encuentro entre el proyecto de una ciencia de las ciencias y la necesaria función expresiva del Yo. En los propios términos de Henrich: ¿cómo puede ser llevado a cabo dicho proyecto, cuando la conciencia misma ha realizado un proceso cognitivo, por medio de la reflexión, y ha convertido al Yo en un Yo-Objeto? ${ }^{6}$

6 La estrategia fichteana parte de la afirmación de que debemos poseer necesariamente un principio fundacional de nuestro saber y de que es posible justificar la posibilidad del mismo en el marco de una ciencia, cuyo principal objetivo sería mostrar su realidad efectiva (Wirklichkeit). Tenemos pues, por un lado, un acercamiento a las intenciones kantianas respecto al procedimiento para justificar la objetividad de nuestro conocimiento - ya que Fichte plantea también la necesidad de trazar la relación entre el desarrollo de la Wissenschaftslehre y la realidad efectiva (Wirklichkeit). Por otro lado, es evidente que esta problematización nos remite al problema de la posibilidad de la postulación de la libertad absoluta, es decir, de la absoluta indeterminación o absoluta expresión del Yo. Bajo estas condiciones el Yo se constituye como el objeto de la investigación.

7 Para Fichte, la fundamentación o búsqueda de la proposición fundamental (Grundsatz), la cual expresaría la certeza de su contenido (Gehalt), es presentada primeramente como un principio de identidad ( $\mathrm{A}=\mathrm{A})$ que explica cómo es posible la autoconciencia, cuyo punto de partida es el arbitrio humano (Willkür). ${ }^{7}$ La justificación debe ser entendida en sentido pragmático, esto es, como la condición de posibilidad real de pensar y postular un principio fundamental que posea certeza inmediata, dicho con otras palabras como el principio básico de este pensar y postular en cuanto acciones concretas de un individuo concreto. El resultado de esta primera postulación es enunciado por Fichte del siguiente modo: 'Si A es, entonces es A'. Esta afirmación no debe ser entendida como un contenido de la proposición, sino como la simple forma del pensar. (cf. GA I, 2, $256 \mathrm{~s}$. GWL) Y continua,

"Por consiguiente, por medio de la afirmación, que la proposición [expresada] más arriba [sc. A =A] es cierta absolutamente, es comprobado que entre aquel si, y este entonces hay una relación necesaria; y la relación necesaria entre ambos es aquella que es puesta sin más y sin fundamento alguno. Llamo a esta relación necesaria provisoriamente $=\mathrm{X} . "(\mathrm{GA} \mathrm{I}, 2,257 \mathrm{GWL})^{8}$

8 Según Fichte, esta $X$ es el resultado del pensamiento del principio de identidad $(A=A)$ desde la Wissenschaftslehre o, expresado de otra manera, la base de todo pensamiento es el principio de identidad. Si queremos formular una proposición fundamental (Grundsatz) tenemos que hacerlo desde supuestos o condiciones ya dadas, externos a nosotros, pero sólo posibles bajo nuestra determinación. Así, dice Fichte, de la misma naturaleza es la Wissenschaftslehre que se pretende fundar, la cual depende de nosotros y no puede existir sin nosotros.

“Así [también es] precisamente nuestra ciencia [ya] descrita. En cuanto tal ella no es algo que exista independiente de nosotros ni sin nuestra intervención, sino que debe ser producida por la libertad de nuestro espíritu actuando [el mismo] siguiendo una dirección determinada". (GA I, 2, 118 BWL) ${ }^{9}$ 
Nosotros hemos de determinar, entonces, la dirección del actuar, del cual esta ciencia debe surgir, y eso es lo que expresa la definición de la WL.

Habría que acentuar, por ello, que hay aquí un aspecto de la teoría que acompaña de manera decisiva la búsqueda de la arriba mencionada proposición fundamental: la libertad absoluta y, con ello, la capacidad del Yo de auto-determinarse y generar un principio indeterminado. Esta fundamentación, cuya expresividad es configurada de manera radical en la teoría de la subjetividad fichteana, no podría ser entendida realmente sin abordar el problema de la libertad absoluta.

III

La disputa sobre la posibilidad de pensar la indeterminación de manera absoluta enfrenta filosóficamente, especialmente si nos remitimos a pasajes kantianos como la dialéctica trascendenltal en su KrV, los sistemas de Fichte y Kant. A diferencia de Kant $^{10}$, Fichte afirma que el acto expresivo del Yo, por medio del cual se ejerce la libertad, se da sólo por medio de una intuición intelectual, pues se cumplen dos condiciones, la de autoconstitución (pues es puesto un Yo actual y potencial) y la de la autoexpresión (en cuanto Yo actuante). Pero cabe señalar que el Yo que se constituye no es una sustancia en sentido pre-crítico, es decir, como sustrato, sino sólo la expresión de la autoconciencia empírica. ${ }^{11}$ Fichte argumenta del siguiente modo la posibilidad de la postulación de un Yo de manera absoluta en su ZE:

"Yo me soy dado por medio de mí mismo, como algo que de cierta manera debe ser activo, con lo cual me soy dado por medio de mí mismo como activo sin más; tengo la vida en mí mismo y la tomo de mí mismo. Sólo a través de este medio de la ley moral me alcanzo a ver; y si me alcanzo a ver a través de esto, me descubro necesariamente como auto-activo. $Y$ por este medio surge para mí ese ingrediente totalmente extraño de la causalidad real de mi Yo [Selbst] en una conciencia que además sería tan sólo la conciencia de una sucesión de mis representaciones." (GA I, $4,219 \mathrm{ZE})^{12}$

Ser capaz de "alcanzar a verse" a través de la ley moral no debe ser tomado literalmente. Lo que parece estar poniendo en juego Fichte aquí es el recurso kantiano de la distinción entre el carácter inteligible y el empírico de una acción. ${ }^{13}$ Si bien es cierto que el acto de auto-ponerse del Yo no constituye sólo un acto empírico, éste tiene además un carácter inteligible, es decir, que no responde a leyes o a una causalidad de la naturaleza, sino a una causalidad según libertad. Este acto de espontaneidad, bajo las consideraciones fichteanas, constituye la prueba para afirmar que la auto-intuición del Yo tiene lugar bajo condiciones establecidas por el idealismo trascendental y no bajo las condiciones empíricas de la experiencia. Es, por ello, una representación del actuar y no de un conocimiento empírico. En estos términos puede ser clarificado de manera más precisa el concepto de expresividad en Fichte.

\section{IV}

El resultado de una intuición intelectual inaugura un nuevo camino filosófico: el desarrollo de una teoría del saber desde la fundamentación de un principio fundamental indeterminado, resultando éste, del acto mismo de auto-ponerse. Dicho de otro modo, podemos considerar que el principio fundamental basado en la libertad de 
indeterminarse posibilita la comprensión de la interacción entre una deducción genética de nuestra estructura cognitiva y la realidad efectiva del mundo. En la medida en que esta teoría de la conciencia desarrolla el carácter expresivo del conocimiento, se retorna a la problemática del Yo, hacia la cual está encaminada gran parte de los esfuerzos de la WL.

El auto-ponerse del Yo es, bajo esta perspectiva, la contraposición del No-Yo, entendido aquí como naturaleza, pero este No-Yo se justifica por el acto mismo del Yo, ya que no es posible delimitar una función expresiva del Yo sin contraponerla a un campo que no depende de nuestro actuar, pero que sólo es posible pensar por medio de nuestra actividad. Visto esto más detenidamente: la proposición Yo $=$ Yo, que remite al principio surgido de la función expresiva y que no significa otra cosa sino la identidad pragmática del Yo, expresa el acto mismo que fundamenta la WL. Pero para que este acto no sea tomado como una mera representación subjetiva, Fichte tiene que postular una segunda proposición fundamental (la de contraposición del No-Yo) y diferenciar así el Yo del No-Yo. Este paso no puede ser dado como una contraposición absoluta, sino relativa al acto del Yo. Dicho de otra manera, hablar de naturaleza o No-Yo implica siempre un acto presente que no puede ser expresado sino desde la Yoidad (Ichheit). Dentro del ámbito abierto por la teoría expresiva del Yo, que el presente trabajo pretende esbozar, es entonces posible comprender la afirmación de Fichte acerca de que la fuente de toda realidad es el Yo (cf. GA I, 143-144 GWL), pues el término 'realidad' debe ser entendido aquí como realidad expresada El objetivo consistiría en demostrar que sólo es posible expresar un contenido (X) si se supone un sujeto que exprese ese contenido X. Suponer la intrusión en una realidad efectiva del No-Yo, sin un sujeto que lo enuncie, implicaría la aceptación de un mundo incognoscible que no se podría explicar desde la unidad de la razón, aquello que Fichte y Kant llamaron la cosa en sí. El Yo pone como realidad absoluta esta diferencia frente al No-Yo.

14 Tomemos el siguiente ejemplo para explicar lo anterior: imaginemos que se traza una línea circular que podríamos denominar A. Nombremos además a la superficie comprendida en la misma ' $\mathrm{X}$ '. Al hacer esta determinación nos oponemos a una superficie infinita, a la cual excluimos en el acto mismo. Si dentro de esta línea quisiéramos trazar otra, digamos ' $\mathrm{Y}$ ', esta dimensión estará comprendida en $\mathrm{A}$, pero se opondrá a la infinitud mencionada y por consiguiente a la dimensión que comprende $\mathrm{X}$. Así, el No-Yo, entendido como una derivación en la Wissenschaftslehre, depende en cuanto tal del Yo, pero no implica que con su postulación se limite la superficie infinita. Este ejemplo demostraría que la WL es la explicación de la relación infinita del postular o trazar una línea (expresión del Yo) y la dimensión infinita de esa superficie (No-Yo):

"Tal como en el espacio las superficies finitas pueden medirse en cuanto a su diferencia, así también, de modo análogo, los grados de realidad, en la medida en que son grados diferentes, pueden medirse en cuanto a su diferencia." ${ }^{14}$

La reflexión, en cuanto método de derivación, sería una herramienta que limita el determinar, pero que concedería la infinitud al No-Yo. De este modo no es difícil ver que la dimensión expresiva del Yo dista mucho de ser considerada como una abstracción o absoluta limitación del No-Yo, sino una función dentro de un sistema complejo.

16 Por lo tanto, la investigación que pretende demostrar la unidad de la conciencia, o dicho mejor, la unidad entre lo teórico y lo práctico adquiere una forma específica e inaugura una nueva teoría de la conciencia o más propiamente, de la autoconciencia. ${ }^{15}$ 
Este método de constitución e investigación, que postula al Yo como objeto y que se justifica por medio de un acto concreto (Ich bin), es lo que prefigura el método reflexivo que más adelante Fichte reemplazará a partir de sus escritos de 1797 con la figura de la intuición intelectual.

Esta nueva teoría de la autoconciencia representa, de este modo, la demostración de que el auto-ponerse del Yo, bajo las condiciones antes mencionadas, produce su propio objeto, es decir, expresa su estructura reflexiva. Constituye, además, la prueba de la unidad de la conciencia y, con ello, la fundamentación de la unidad de lo subjetivo y lo objetivo. Esto es justamente lo que podría denominarse teoría expresiva del Yo. La misma tendría como principal objetivo definir la relación entre el Yo que se concretiza en su impulso hacia lo indeterminado y su desarrollo dentro de su propia actividad.

\section{NOTAS}

1. En este sentido el proyecto fichteano comparte su intención con el de K. L. Reinhold expresado en su Versuch einer neuen Theorie des menschlichen Vorstellungsvermögen. Prag-Jena 1789. (Cf. J. STOLZENBERG, ,"Geschichte des Selbstbewusstseins'. Reinhold-Fichte-Schelling“, en: Internationales Jahrbuch des Deutschen Idealismus. 1, 2003: 93-113)

2. Estos supuestos están basados en la reconstrucción del $\S 1$ de la GWL realizada por J. Stolzenberg. En su artículo „Fichtes Satz »Ich bin«. Argumentanalytische Überlegungen zu Paragraph 1 der Grundlage der gesamten Wissenschaftslehre von 1794/95“, en: F-S 6, 1994: 1-34. En ella Stolzenberg muestra los distintos tránsitos desde el principio de identidad $(\mathrm{A}=\mathrm{A})$ a la postulación de un Yo constituido como agente normativo (Ich bin) en el § 1 de GWL.

3. Ciertamente en estudios actuales como el de R. BRANDOM (Articulating Reasons: An Introduction to Inferentialism. Cambridge 2000) se ha introducido el término ' expresividad' como una función subjetiva que hace posible dar razones y hacer inferencias. La intención aquí es, sin embargo, analizar sólo el papel que juega la función expresiva del Yo dentro a la teoría de la subjetividad fichteana.

4. Cf. F. BEISER, German Idealism. The Struggle against subjectivism, 1781-1801. Cambridge 2002.

5. Lo que queda claro en esta comparación es que a Fichte le interesa de manera fundamental la capacidad de un Yo que se auto-pone y, desde esa posibilidad, poder explicar la relación entre lo subjetivo y lo objetivo. F. Beiser menciona a propósito de las condiciones arriba mencionadas: "to be self-positing implies self-constitution since the self-knowing self brings itself into existence when it posits itself; there is no pre-existing self who is just given prior to the self-knowing self. Self-positing also implies self-expression since to say that the self posits itself as $P$ is to say that the self simply is $P$, that it makes itself what it is through $P$ or whatever it posits itself to be." (F. BEISER, op. cit. 304)

6. Cf. D. HENRICH, „Fichtes ursprüngliche Einsicht“, en: Subjektivität und Metaphysik. Frankfurt am Main 1966: 195. 
7. Cabe recordar que la recepción post-kantiana y hegeliana de la filosofía crítica retoma de manera importante la noción de Willkür, concepto que adquiere un rol importante en la formulación de sus sistemas.

8. „Mithin wird durch die Behauptung, daß der obige Satz schlechthin gewiß sei, das festgestellt, daß zwischen jenem Wenn, und diesem So ein notwendiger Zusammenhang sei; und der notwendige Zusammenhang zwischen beiden ist es, der schlechthin, und ohne allen Grund gesetzt wird. Ich nenne diesen notwendigen Zusammenhang vorläufig = X."

9. Trad. E. Acosta. „So unsere eben beschriebene Wissenschaft. Sie ist als solche, nicht etwas, das unabhängig von uns, und ohne unser Zuthun existiere, sondern das erst durch die Freiheit unsers nach einer bestimmten Richtung hin wirkenden Geistes hervorgebracht werden soll.“

10. Para su confrontación, véase la antinomia de la libertad y los paralogismos de la razón pura. Ahí Kant intenta refutar la idea de la postulación de un Yo pienso vacío capaz de constituír algún tipo de conocimiento. (KrV. A 444 / B 472 y A 349 s.)

11. Cf. F. BEISER. op. cit. 304. Véase además especialmente el capítulo II.

12. „ich werde mir durch mich selbst als etwas, das auf eine gewisse Weise thätig sein soll, gegeben, ich werde mir sonach durch mich selbst als tätig überhaupt gegeben; ich habe das Leben in mir selbst, und nehme es aus mir selbst. Nur durch dieses Medium des SittenGesetzes erblicke ich mich; und erblicke ich mich dadurch, so erblicke ich mich nothwendig, als selbstthätig; und dadurch entsteht mir das ganz fremdartige Ingrediens der reellen Wirksamkeit meines Selbst in einer Bewußtsein, das außerdem nur das Bewußtsein einer Folge meiner Vorstellungen sein würde."

13. Cf. KrV, B $570-586$.

14. R. Lauth presenta esta analogía de manera adecuada, pues deja claro que la síntesis de las proposiciones 'Yo = Yo' y 'Yo no es = No-Yo' se expresa como la infinita dimensión del No-Yo, pero sin dejar de acentuar que sólo el yo puede dar lugar a dicha reflexión. Véase, R. LAUTH, La doctrina trascendental de la naturaleza de Fichte según los principios de la Doctrina de la ciencia. Madrid 1999: 52

15. Véase D. HENRICH, op. cit. Henrich enfatiza especialmente la nueva forma de la autoconciencia bajo el esquema fichteano.

\section{RESÚMENES}

In what follows I will deal with a specific function assigned to the Self (Ich) in the first paragraph of Fichte's Foundation of the Entire Doctrine of Knowledge. I will propose that this function can be characterized as an expressive one that refers to the self-undetermined position of the Self. Firstly, I will argue that this undeterminacy is necessary for the development of Fichte's philosophical project. Then I will suggest that this expressive function shows the philosophically radical nature of subjectivity. In doing so I attempt at making clear the importance of the selfexpression of the Self in the development of Fichte's theory of subjectivity.

\section{ÍNDICE}

Keywords: Foundation of the Entire Doctrine of Knowledge (GWL), expression, self, subjectivity 
AUTOR

GUSTAVO MACEDO RODRÍGUEZ

Halle/Saale 